\title{
Multimodal Imaging of Cation Disorder and Oxygen Deficiency-Mediated Phase Separation in Double Perovskite Oxides
}

\author{
Steven R. Spurgeon ${ }^{1}$, Peter V. Sushko ${ }^{1}$, Arun Devaraj ${ }^{1}$, Yingge Du ${ }^{1}$, Timothy Droubay ${ }^{1}$, and Scott A. \\ Chambers ${ }^{1}$ \\ 1. Physical and Computational Sciences Directorate, Pacific Northwest National Laboratory, Richland, \\ Washington, USA
}

Extensive work over the past decade has detailed the mechanisms governing the polar discontinuity in oxide heterostructures, greatly improving our understanding of emergent interface properties. However, less attention has focused on how the polar discontinuity affects synthesis pathways and phase separation, limiting us from realizing many predicted thin film structures. A new multiscale analysis approach, leveraging state-of-the-art characterization techniques and advanced theory calculations, is needed to untangle the complex interplay of factors that govern oxide thin film synthesis.

Here we describe a novel cation disorder and oxygen deficiency-mediated phase separation mechanism in the complex oxide, $\mathrm{La}_{2} \mathrm{MnNiO}_{6}$ (LMNO), using a correlative analysis via aberration-corrected scanning transmission electron microscopy (STEM) and atom probe tomography (APT). High-angle annular dark field (STEM-HAADF) and annular bright field (STEM-ABF) imaging show that a distinct, quasi-epitaxial relationship is preserved between the precipitates and matrix, even tens of nanometers away from the substrate (see Figure 1). However, in contrast to previous measurements of $\mathrm{LaAlO}_{3} /$ $\mathrm{LaNiO}_{3}$ thin films [1], APT measurements (see Figure 2) reveal a complex distribution of precipitates that nucleate several nanometers away from the substrate. Our multiscale analysis indicates the presence of a phase-pure interface layer, upon which extensive phase separation into ordered LMNO and NiO precipitates occurs.

While we have previously shown that phase separation is thermodynamically favorable in low oxygen fugacity [2], the actual mechanism of separation is unknown. Here ab initio simulations lead us to propose a two-step growth process to interpret this curious behavior. During the initial growth stage, LMNO films are ordered, although we cannot rule out the formation of isolated point defects; as growth progresses, an increasing internal electric field due to the polar discontinuity promotes the formation of surface oxygen vacancies and associated Ni-rich precipitates. Our study blends experiment and theory, highlighting a new method to control oxide phase separation via the coupling between cation disorder and the polar discontinuity. 
References:

[1] Detemple, E. et al. Polarity-driven nickel oxide precipitation in $\mathrm{LaNiO}_{3}-\mathrm{LaAlO}_{3}$ superlattices. Appl. Phys. Lett. 99, 211903 (2011).

[2] Spurgeon, S. R. et al. Competing pathways for nucleation of the double perovskite structure in the epitaxial synthesis of $\mathrm{La}_{2} \mathrm{MnNiO}_{6}$. Chem. Mater. 28, 3814-3822 (2016).

[3] This work was supported by the U.S. Department of Energy, Office of Science, Division of Materials Sciences and Engineering under award \#10122.
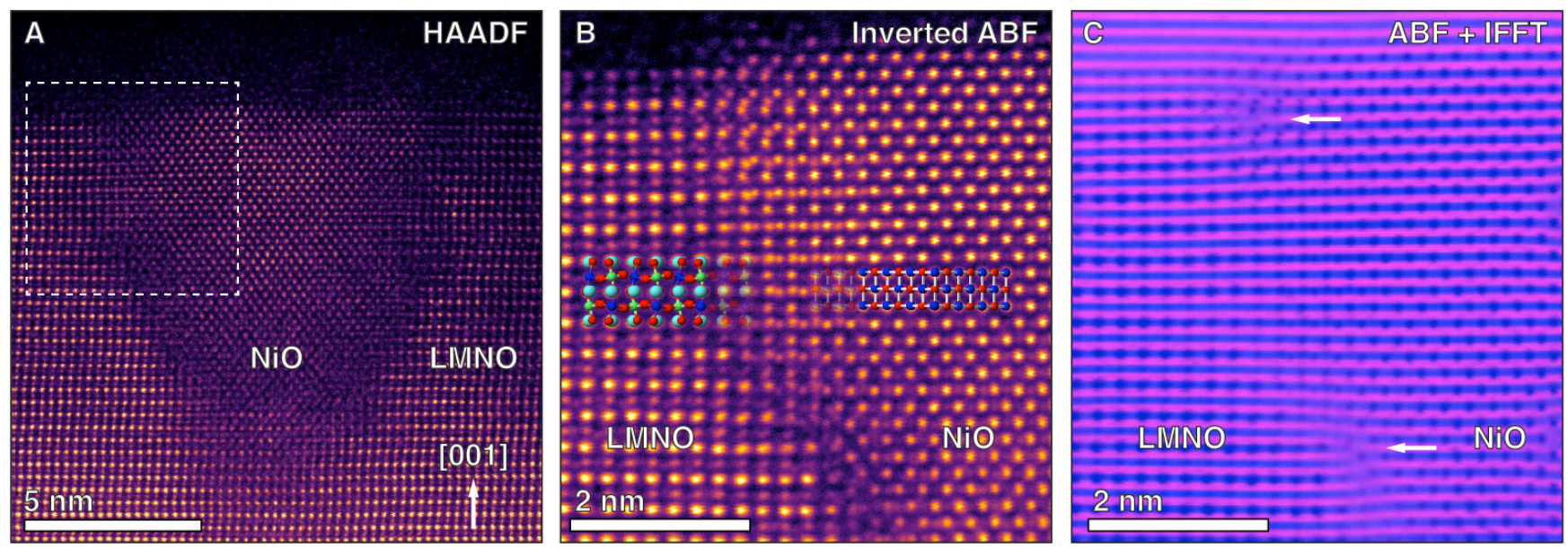

Figure 1: STEM imaging of the precipitate / matrix interface. (A) Representative STEM-HAADF cross-section of a thin region containing a faceted $\mathrm{NiO}$ precipitate and the surrounding LMNO matrix. (B) Inverted, drift-corrected STEM-ABF image revealing the distinct orientation relationship between the matrix and precipitate. Theoretical crystal structures are overlaid onto the image. (C) Inverse, masked fast Fourier transform (IFFT) overlaid on the STEM-ABF image to highlight the presence of misfit dislocations that accommodate the out-of-plane lattice parameter mismatch (marked by arrows).
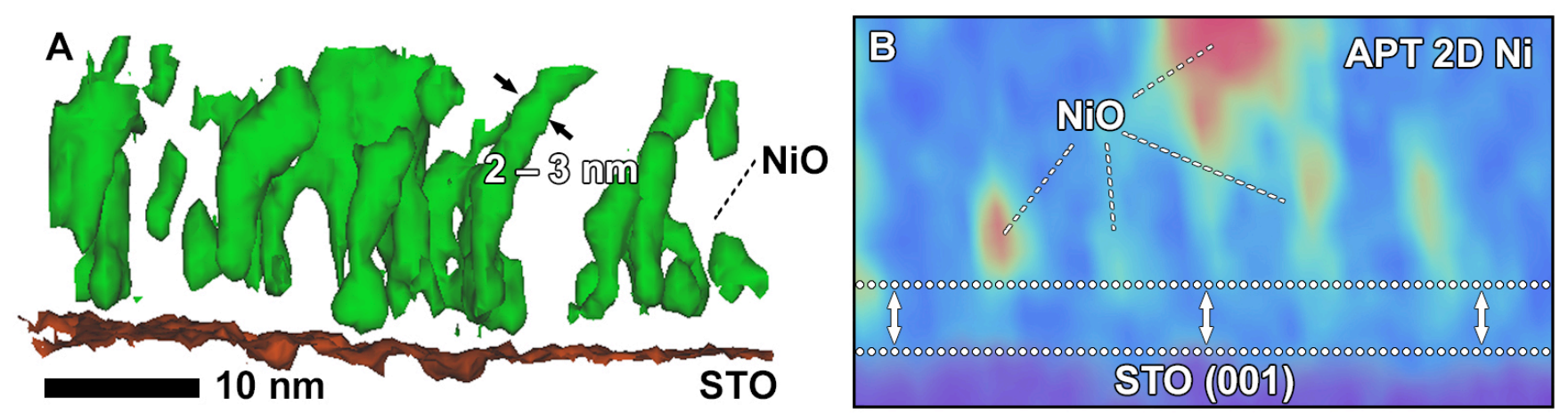

Figure 2: Correlative APT mapping of NiO precipitate morphology and distribution. (A) Oblique view of a 15 at $\% \mathrm{Ni}$ iso-surface of the sample, revealing faceted $\mathrm{NiO}$ precipitates marked by arrows. Volume: $1 \mathrm{~nm} \times 30 \mathrm{~nm} \times 40 \mathrm{~nm}$. (B) 2D Ni composition map from the dataset in (A), highlighting the gap between precipitates and the substrate (marked by arrows). 